\title{
Associação entre a qualidade de vida e o estresse em estudantes de Fonoaudiologia
}

\section{Association between quality of life and stress in speech, language, and hearing sciences students}

\author{
Kelly da Silva' (D), Vanessa Veis Ribeiro' ${ }^{10}$, Carla Patrícia Hernandez Alves Ribeiro César ${ }^{2}$ (D), \\ Alicélia Soares Oliveira ${ }^{1}$ (1) , Rodrigo Dornelas ${ }^{3}$ (D), Patrícia PinattiMoreira ${ }^{4}$, Raphaela Barroso Guedes Granzotti ${ }^{2}$
}

\section{RESUMO}

Objetivo: Verificar a associação entre a qualidade de vida - a partir dos domínios "bem-estar físico e material"; "relações com outras pessoas"; "atividades sociais, comunitárias e cívicas"; "desenvolvimento pessoal e realização" " "recreação"-, com o estresse em estudantes de Fonoaudiologia. Métodos: Quarenta estudantes (34 do gênero feminino), de um curso de graduação em Fonoaudiologia, com idades entre 19 e 39 anos, responderam às questões da Escala de Qualidade de Vida de Flanagan e do Inventário de Sintomas de Stress para Adultos de Lipp e os resultados foram analisados por meio de regressão logística binária. Resultados: Os domínios relacionados à qualidade de vida com piores resultados foram "bem-estar físico e material" e "desenvolvimento pessoal e realização" e o domínio que apresentou melhores resultados foi "relações com outras pessoas", sendo capaz de prever 21,2\% dos casos de estresse. A qualidade de vida no domínio "relações com outras pessoas" foi uma preditora significativa do estudo, sendo considerada como protetora para o estresse na amostra investigada. Conclusão: Uma boa qualidade de vida no domínio "relações com outras pessoas" apresentou-se como fator protetor do estresse em estudantes do curso de Fonoaudiologia.

Palavras-chave: Qualidade de vida; Indicadores de qualidade de vida; Estresse psicológico; Saúde do estudante; Estudantes

\begin{abstract}
Purpose: To verify the association between quality of life - from the physical and material well-being domains; relationships with other people; social, community and civic activities; personal development; achievement and recreation -, with stress in Speech, Language, and Hearing Sciences. Methods: 40 students (34 females) from an undergraduate course in Speech, Language, and Hearing Sciences, aged between 19 and 39, answered the Flanagan Quality of Life Scale and Lipp's Stress Symptoms Inventory. The results were analyzed using binary logistic regression. Results: The domains related to the quality of life with the worst results were "physical and material well-being" and "personal development and achievement," and the domain that showed the best results were "relationships with other people", being able to predict $21.2 \%$ of the cases of stress. The quality of life in the "relationships with other people" domain was a significant predictor of the study, being considered as protective for stress in the investigated sample. Conclusion: A good quality of life in the domain "relationships with other people" was a protective factor of stress in students of the Speech, Language, and Hearing Sciences.
\end{abstract}

Keywords: Quality of life; Indicators of quality of life; Psychological stress; Student health; Students

\footnotetext{
Trabalho realizado no Departamento de Fonoaudiologia - DFOL, Universidade Federal de Sergipe - UFS - Lagarto (SE), Brasil.

${ }^{1}$ Departamento de Fonoaudiologia - DFOL, Universidade Federal de Sergipe - UFS - Lagarto (SE), Brasil.

${ }^{2}$ Departamento de Fonoaudiologia - DFO, Universidade Federal de Sergipe - UFS - São Cristóvão (SE), Brasil.

${ }^{3}$ Departamento de Fonoaudiologia, Universidade Federal do Rio de Janeiro - UFRJ - Rio de Janeiro (RJ), Brasil.

${ }^{4}$ Psicóloga Clínica - Aracaju (SE), Brasil

Conflito de interesses: Não.

Contribuição dos autores: KS concepção do estudo, interpretação dos dados, redação e revisão do manuscrito; VVR análise dos dados, revisão final do manuscrito; CPHARC interpretação dos dados e revisão final do manuscrito; ASO coleta e interpretação dos dados, revisão final do manuscrito; RD interpretação dos dados, redação e revisão final do manuscrito; PPM análise do Inventário de estresse e revisão final do manuscrito; RBGG concepção do estudo, coleta e interpretação dos dados, redação e revisão final do manuscrito; coordenadora do projeto.
}

Financiamento: Nada a declarar.

Autor correspondente: Kelly da Silva. E-mail: kelly.fonoufs@gmail.com

Recebido: Fevereiro 03, 2021; Aceito: Outubro 11, 2021 


\section{INTRODUÇÃO}

Na graduação, os estudantes vivenciam transformações que variam em função de fatores relacionados à dificuldade de adaptação às novas demandas. Isso pode afetar a motivação e a autoestima, além de gerar estresse e impactar a qualidade de vida $(\mathrm{QV})^{(1-3)}$.

Estudantes universitários apresentam alta ocorrência de estresse $^{(1,3,4)}$, que pode ser definido como a busca do organismo para se adaptar frente a uma situação percebida como ameaçadora à sua vida e à sua homeostase interna ${ }^{(5)}$. Alguns aspectos são comuns à transição enfrentada na fase universitária, como a expectativa com a carreira, a necessidade de muitas horas de estudo e as relações interpessoais. Entretanto, há fatores associados às diferenças individuais, sociais e econômicas ${ }^{(6)}$ que podem contribuir para o surgimento ou agravamento do estresse, condição esta que pode comprometer o desempenho acadêmico, afetar a saúde e gerar sensações de incapacidade nos discentes ${ }^{(4)}$.

Estudos apontam existência de relação entre a presença de estresse e a qualidade de vida em estudantes universitários ${ }^{(1)}$, demonstrando que, quanto maior a $\mathrm{QV}$ dos estudantes, menores os níveis de estresse. A qualidade de vida pode ser definida como a percepção que o indivíduo tem de sua posição na vida, no contexto da cultura, no sistema de valores em que vive e em relação aos seus objetivos, expectativas, padrões, preocupações e desejos ${ }^{(7)}$.

Diante da complexidade que é a prevenção de estresse em universitários e sabendo-se que o estresse e a qualidade de vida podem estar associados, faz-se necessário verificar se há domínios da QV relacionados ao estresse dos discentes, sobretudo nos discentes de Fonoaudiologia. Tais informações são muito importantes para direcionar o trabalho com saúde mental desses futuros profissionais.

Além disso, são escassos os estudos brasileiros que tratam desse tema. Pesquisadores ${ }^{(8)}$ verificaram, pela utilização do instrumento WHOQOL-bref (The World Health Organization Quality of Life), que estudantes de Fonoaudiologia apresentaram, de forma geral, boa QV. Os domínios que indicaram bons escores foram saúde e relações sociais, enquanto o que representou menor escore foi o meio ambiente. Outros ${ }^{(9)}$ verificaram alto índice de estresse em estudantes de Fonoaudiologia. Desta forma, o enfrentamento do estresse, a fim de propiciar melhor QV ao acadêmico de Fonoaudiologia, mostra-se relevante nos dias atuais, conforme apontado na literatura ${ }^{(10)}$. No entanto, estudos com foco em estudantes de Fonoaudiologia, que analisem as duas variáveis em conjunto (QV e estresse) não foram publicados até o momento, ratificando a importância desta pesquisa.

Considerando a importância do tema, o objetivo deste estudo foi verificar a associação entre a qualidade de vida - a partir dos domínios bem-estar físico e material; relações com outras pessoas; atividades sociais, comunitárias e cívicas; desenvolvimento pessoal; realização e recreação -, com o estresse em estudantes de Fonoaudiologia.

\section{MÉTODOS}

Estudo observacional, transversal e analítico. O estudo foi aprovado pelo Comitê de Ética em Pesquisa da Universidade Federal de Sergipe (parecer $\mathrm{n}^{\circ}$ 2.377.894). A coleta de dados foi realizada no interior de um estado nordestino, finalizando em 2019. Foram recrutados discentes de um curso de Fonoaudiologia, com idade superior a 18 anos, que assinaram o Termo de Consentimento Livre e Esclarecido. Foram excluídos participantes que deixaram os instrumentos incompletos e que estavam cursando o primeiro ano do curso. Justifica-se a exclusão de primeiranistas, tendo em vista que estudantes das séries iniciais podem apresentar pródromos de ansiedade, depressão e dificuldades em relação à ajuda, inerentes à adaptação à vida universitária ${ }^{(11)}$, evitando-se, portanto, esse viés de análise.

O cálculo do tamanho da amostra foi realizado por meio de teste intervalo de confiança para a média. Consideraram-se os seguintes parâmetros: nível de confiança de $95 \%$, margem de erro de $5 \%$ e desvio padrão estimado da população de estudantes de Fonoaudiologia da Universidade Federal de Sergipe do Campus de Lagarto, para a variável independente de 14,9. Considerou-se esse desvio padrão por ser o maior apresentado para a qualidade de vida. $\mathrm{O}$ tamanho estimado da amostra foi de 37 participantes. Após aplicação dos critérios de elegibilidade, a amostra final do presente estudo foi composta por 40 graduandos de Fonoaudiologia.

A coleta de dados foi realizada por meio da autorresposta dos dados de identificação, preenchimento da Escala de Qualidade de Vida de Flanagan (EQVF), proposta por Flanagan ${ }^{(12)}$ nos Estados Unidos, traduzida e validada para o português brasileiro ${ }^{(13)}$ e do Inventário de Sintomas de Stress para Adultos de Lipp (ISSL), seguindo as indicações de aplicação e análise dos autores para divisão dos discentes em "com estresse" e "sem estresse".

Os universitários participantes tinham entre 19 e 39 anos (média de 25 $\pm 5,18$ ), sendo mais frequentes os do gênero feminino $(n=34,85 \%)$, solteiros $(n=31,77,5 \%)$, que não exerciam função remunerada $(n=33,82,5 \%)$, residiam com familiares $(n=22$, $55 \%)$ e que se mudaram de cidade devido ao curso $(n=18,45 \%)$.

A variável independente foi a QV e avaliada pela EQVF. O instrumento é composto por 15 questões, respondidas em uma escala Likert de 7 graus, em que "1" indica muito insatisfeito e "7", muito satisfeito; desta forma, o escore total pode variar de 15 a 105 pontos. Foram analisados os domínios "bem-estar físico e material"; "relações com outras pessoas"; "atividades sociais, comunitárias e cívicas"; "desenvolvimento pessoal e realização"; "recreação" e o escore total.

A variável dependente foi a presença de estresse. Para identificar a presença de estresse, foi aplicado o ISSL ${ }^{(14)}$. O instrumento é composto por 55 itens, para os quais os participantes devem assinalar os sintomas que têm experimentado em determinado período (últimas 24 horas, última semana e último mês) e foi analisado por uma autora psicóloga. Utilizou-se apenas a presença ou ausência de estresse.

Empregou-se a análise de regressão logística binária e o cálculo da odds ratio para verificar se os domínios de QV estavam associados ao estresse. No primeiro modelo ajustado, os coeficientes das variáveis "bem-estar físico e mental" "atividades sociais, comunitárias e cívicas", "desenvolvimento pessoal e realização" e "recreação" não foram significativos. Assim, foi ajustado um modelo sem essas variáveis. Adotou-se o nível de significância de 5\% e software utilizado foi o SPSS 25.0.

\section{RESULTADOS}

A maioria dos estudantes apresentou estresse $(n=34,85 \%)$. A Tabela 1 mostra a caracterização das variáveis independentes referentes aos domínios de QV. O domínio que apresentou melhores resultados referentes à qualidade de vida foi "relações com outras pessoas" (Tabela 1).

A análise de regressão resultou em um modelo estatisticamente significativo, no qual o domínio "relações com outras pessoas" foi capaz de prever $21,2 \%$ da variabilidade da variável estresse $\left[X^{2}(1) p=0,023 ; R^{2}\right.$ Nagelkerke $\left.=0,212\right]$. A QV no domínio 
Tabela 1. Análise descritiva dos domínios de qualidade de vida em estudantes de Fonoaudiologia

\begin{tabular}{lccccccc}
\hline \multicolumn{1}{c}{ Domínios } & Média & DP & Mínimo & Máximo & 1Q & Mediana & $\mathbf{3 Q}$ \\
\hline Bem-estar físico e material & 4,8 & 1,5 & 1,5 & 7,0 & 4,0 & 5,5 & 6,0 \\
Relações com outras pessoas & 5,3 & 1,0 & 2,3 & 6,8 & 4,9 & 5,5 & 6,3 \\
Atividades sociais, comunitárias e cívicas & 5,2 & 1,0 & 2,0 & 7,0 & 4,9 & 5,3 & 6,0 \\
Desenvolvimento pessoal e realização & 4,8 & 1,2 & 1,5 & 7,0 & 4,4 & 5,0 & 5,6 \\
Recreação & 5,1 & 1,4 & 1,7 & 7,0 & 4,6 & 5,3 & 6,0 \\
Total & 75,8 & 14,9 & 36,0 & 102,0 & 69,0 & 78,5 & 84,5 \\
\hline
\end{tabular}

Legenda: $1 \mathrm{Q}=$ primeiro quartil; $3 \mathrm{Q}=$ terceiro quartil; $\mathrm{DP}=$ desvio padrão

Tabela 2. Análise dos fatores preditivos do estresse

\begin{tabular}{|c|c|c|c|c|c|c|c|c|}
\hline & \multirow{2}{*}{ B } & \multirow{2}{*}{ S.E. } & \multirow{2}{*}{ Wald } & \multirow{2}{*}{ df } & \multirow{2}{*}{ valor de $p$} & \multirow{2}{*}{ OR } & \multicolumn{2}{|c|}{ IC 95\% para OR } \\
\hline & & & & & & & Inferior & Superior \\
\hline Relações com outras pessoas & $-1,375$ & 0,748 & 3,384 & 1 & 0,066 & 0,253 & 0,058 & 1,094 \\
\hline Constante & 9,592 & 4,504 & 4,536 & 1 & 0,033 & & & \\
\hline
\end{tabular}

Modelo de regressão logística binária

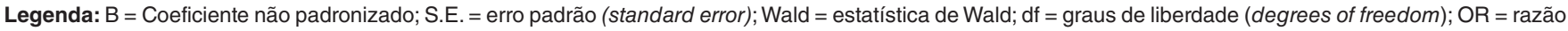
de chances (odds ratio); IC = intervalo de confiança

"relações com outras pessoas" foi um fator de proteção do estresse $(\mathrm{OR}=0,253$; IC 95\% =0,058 - 1,094). A chance de estresse em pessoas com boa QV no domínio "relações com outras pessoas" foi $25,3 \%$ menor do que em pessoas com ausência dessa condição. Os resultados estão apresentados na Tabela 2.

\section{DISCUSSÃO}

Este estudo pretendeu verificar a associação entre a qualidade de vida e a presença de estresse em estudantes de Fonoaudiologia, elencando possíveis fatores protetores e/ou fatores de risco para a presença de estresse. Esses aspectos têm sido explorados na literatura em razão da sua forte influência no desempenho acadêmico dos estudantes ${ }^{(2)}$. Entretanto, estudos que discutam à luz da literatura esses achados em estudantes de Fonoaudiologia são escassos. Pesquisadores brasileiros ${ }^{(15)}$ compararam a qualidade de vida de estudantes dos cursos de Medicina e Fonoaudiologia, concluindo que, nos domínios psicológico e físico, os estudantes de Fonoaudiologia apresentaram menores escores, porém, melhores resultados na percepção do ambiente de ensino, bem como em equilibrar compromissos (pessoais e acadêmicos), em especial, nas séries finais do curso. Tais resultados apontam, segundo os pesquisadores, para a necessidade de maior atenção para esse grupo de estudantes.

Neste estudo, os domínios com piores resultados foram "bem-estar físico e material" - relacionado à disposição física, conforto e tranquilidade - e "desenvolvimento pessoal e realização", que expressa autoaceitação, autonomia, senso de domínio, boa relação com os outros e procura por metas ${ }^{(16)}$. As melhores respostas por domínio foram observadas no domínio "relações com outras pessoas". Esses dados concordam com os achados de um estudo sobre QV de estudantes de Nutrição, que obtiveram melhores resultados também nesse domínio ${ }^{(17)}$.

Os resultados obtidos neste estudo, por meio do ISSL indicaram que $85 \%$ dos estudantes apresentaram estresse. Estudo com discentes de diferentes cursos da área da saúde ${ }^{(3)}$, utilizando o ISSL para aferição do estresse, mostrou ocorrência de estresse inferior ao do presente estudo. Um dos fatores que pode ter contribuído para este achado foi a presença majoritária de mulheres na amostra, pois há fortes indícios na literatura que as mulheres estão mais suscetíveis ao estresse ${ }^{(18)}$.

Ficou evidenciado que uma boa QV no domínio "relações com outras pessoas" foi um fator de proteção para o estresse, ou seja, estudantes que conseguem estabelecer boas relações pessoais e profissionais têm maior chance de não apresentar estresse. Cabe ressaltar que os participantes desta pesquisa eram discentes de Fonoaudiologia, em que a comunicação e a interação social são discutidas durante todo o curso. Portanto, estudar esse domínio e sua influência na qualidade de vida pode facilitar o conhecimento de desafios e facilitadores no manejo do estresse, sendo as relações pessoais possivelmente um potente facilitador.

Estudo brasileiro que objetivou investigar o efeito do Treinamento de Habilidades Sociais (THS) nas habilidades sociais e nos níveis de estresse de estudantes universitários concluiu que essas habilidades foram capazes de diminuir significativamente o estresse entre os participantes da pesquisa ${ }^{(19)}$. Estes achados concordam e justificam o fato de que, na presente pesquisa, um bom escore referente ao relacionamento com outras pessoas apresentou-se como protetor para o estresse.

Faz-se importante discutir outras variáveis relacionadas ao presente estudo, dentre as quais destacam-se: gênero, atividade laboral, estado civil e local de residência dos participantes da amostra. Em relação ao gênero, a amostra foi composta, com maior frequência, pelo feminino, ratificando o exposto na literatura ${ }^{(20)}$, de que, embora nas últimas décadas, venha ocorrendo aumento de ingressantes do gênero masculino no curso de Fonoaudiologia, a maioria ainda é do gênero feminino. Além disso, há evidências de que o estresse impacta diferentemente os gêneros, tendo em vista que autoras ${ }^{(21)}$ relataram estresse nos homens quando ocupavam posições de chefia ou eram profissionais liberais e, nas mulheres, quando ocupavam cargos de escritório ou eram acadêmicas, além de menor estresse quando o estado civil era de solteiro, concordando com os dados obtidos na presente pesquisa, uma vez que a maioria da amostra não exercia função remunerada e era solteira.

Já em relação à moradia, outras condições de vida (tais como a situação de vida na infância, o relacionamento do estudante com sua família, suas condições socioeconômicas, entre outras) impactam a saúde mental e a qualidade de vida estudantil(11), 
limitando uma análise mais aprofundada sobre o assunto, levando em conta que essas condições não foram informadas. No entanto, a maioria residia com a família, o que pode favorecer a melhor qualidade de vida, pois os rituais familiares afetam positivamente, tanto a qualidade de vida quanto a relacional ${ }^{(22)}$, ratificando a importância da "relação com outras pessoas" como fator protetor do estresse e, consequentemente, melhor QV.

Os resultados obtidos alertam para a necessidade de ações universitárias voltadas para a saúde mental de discentes e que enfatizem a importância das relações sociais. $\mathrm{O}$ bem-estar do estudante influencia diretamente a sua formação, pois interfere no planejamento, na organização e na atenção aos estudos ${ }^{(2)}$.

O presente estudo, na forma de comunicação breve, apresenta como limitação o fato de ter sido desenvolvido apenas com estudantes de Fonoaudiologia que utilizavam metodologias ativas de ensino, o que dificultou a extrapolação dos resultados. Sendo assim, novos estudos têm sido desenvolvidos pela equipe, ampliando o público-alvo.

Independentemente dessa limitação, sugere-se que o enfrentamento do estresse em estudantes de Fonoaudiologia possa ser norteado por ações promotoras de QV, associadas às relações sociais saudáveis. $\mathrm{O}$ saber-fazer e o saber-viver precisam coexistir harmonicamente para que o desempenho acadêmico não entregue ao mercado de trabalho profissionais vulneráveis ao adoecimento.

\section{CONCLUSÃO}

Uma boa qualidade de vida no domínio "relações com outras pessoas" apresentou-se como fator protetor do estresse em estudantes do curso de Fonoaudiologia.

\section{REFERÊNCIAS}

1. Alfredo PP, Biondi JCL, Manna V. Evaluation of quality of life and stress in academic course of Physiotherapy. J Heal Sci Inst. 2016;34(4):22430.

2. Silva PCS, Sousa JCM, Nogueira ER, Sousa MNA, Assis EV, Dias MJ. Physiotherapy course students life quality and health conditions. Rev Bras Educ Saúde. 2019;9(4):10-7.

3. Murakami K, Panúncio-Pinto M, Santos J, Troncon L. Psychological stress in students from undergraduate courses in health professions: contribution to promote mwntal health. Rev Med. 2019;98(2):108-13. http://dx.doi.org/10.11606/issn.1679-9836.v98i2p108-113.

4. Kam SXL, Toledo ALS, Pacheco CC, Souza GFB, Santana VLM, Bonfá-Araujo B, et al. Stress in undergraduate medical students. Rev Bras Educ Med. 2019;43(1, Supl. 1):246-53. http://dx.doi. org/10.1590/1981-5271v43suplemento1-20180192.

5. Moreira TDS, Araújo GF. Percepção de estresse entre discentes do curso de enfermagem de uma IES. Id Line Rev Psicol. 2017;11(35):479-91. http://dx.doi.org/10.14295/idonline.v11i35.750.

6. Nogueira-Martins LA, Nogueira-Martins MCF. Saúde mental e qualidade de vida de estudantes universitários. Rev Psicol Divers e
Saúde. 2018;7(3):334-7. http://dx.doi.org/10.17267/2317-3394rpds. v7i3.2086.

7. Whoqol Group. Development of the WHOQOL: rationale and current status. Int J Ment Health. 1994;23(3):24-56. http://dx.doi.org/10.108 0/00207411.1994.11449286.

8. Raquel ACS, Kuroishi RCS, Mandrá PP. Quality of life of students in Speech, Language and Hearing Sciences. Rev CEFAC. 2016 Out;18(5):1133-40. http://dx.doi.org/10.1590/1982-021620161853916.

9. Guedes-Granzotti RB, Dornelas R, Galdino MKVR, Leite IS, Oliveira PF, Moreira PP, et al. Estresse discente em um curso de Fonoaudiologia. Audiol Commun Res. 2021;26:e2335. http://dx.doi.org/10.1590/23176431-2020-2335.

10. Albuquerque JDS, Silva DD, Cardozo FNH, Silva RKR, Souza PMB, Silva AFM, et al. Use of integrative and complementary practices as a resource to reduce levels of anxiety and stress in Speech Therapy students. Braz J Heal Rev. 2020;3(5):11461-72. http://dx.doi. org/10.34119/bjhrv3n5-008.

11. Osse CMC, Costa II. Mental health and quality of life at a student hall of residence at the Universidade de Brasília, Brazil. Estud Psicol [Internet]. 2011 Mar [citado em 2021 Fev 3];28(1):115-22. Disponível em: http://www.scielo.br/scielo.php?script=sci_arttext\&pid=S0103166X2011000100012\&lng=pt\&tlng=pt

12. Flanagan JC. Measurement of quality of life: current state of the art. Arch Phys Med Rehabil. 1982;63(2):56-9. PMid:6460487.

13. Nasser L, Gonçalves LHT. Avaliação de uma escala de medida de qualidade de vida. Texto Contexto Enferm. 1999;8(3):99-110.

14. Lipp MEN. Manual do inventário de sintomas de stress para adultos de Lipp. São Paulo: Casa do Psicólogo; 2005.

15. Tempski PZ, Martins M A, Silveira PSP, Garcia VL, Perotta B. Qualidade de vida do estudante. In: Marchesan IQ, Justino H, Tomé MC, editores. Tratado das especialidades em fonoaudiologia. São Paulo: Guanabara Koogan; 2014. p. 1101-5.

16. Silva PC, Mota MS, Neutzling A, Amorim CB, Blan B S, Oliveira SM. Instruments for the evaluation of quality of life of people with intestinal stomies: integration review. Rev Enferm Atual. 2019;90(28):1-8.

17. Leite ACB, Grillo LP, Caleffi F, Mariath AB, Stuker H. Qualidade de vida e condições de saúde de académicos de nutrição. Rev Saúde Pública do Paraná. 2011;13(1):82-90.

18. Lameu JN, Salazar TL, Souza WF. Prevalence of stress symptoms among students of a public university. Rev Psicol Educ. 2016;42(42):13-22.

19. Nazar TCG, Pering AC, Girotto DA, Kucmanski T. Treinamento de habilidades sociais em universitários: um estudo acerca das variáveis estresse e habilidades sociais. Rev Educ Psicol e Interfaces. 2020;4(1):190204. http://dx.doi.org/10.37444/issn-2594-5343.v4i1.210.

20. Santos ACM, De Luccia G. Profile of speech therapy students according to the National Survey of Student Performance. Distúrb Comun. 2015;27(3):589-99.

21. Sadir MA, Bignotto MM, Lipp MEN. Stress and quality of life: the influence of some personal variables. Paid. 2010;20(45):73-81. http:// dx.doi.org/10.1590/S0103-863X2010000100010.

22. Silva ACR. A relação entre o conflito trabalho-família, a qualidade de vida e a qualidade relacional: qual o papel dos rituais familiares? [dissertação]. Coimbra: Universidade de Coimbra; 2014. 